\title{
Contaminación ambiental por mercurio y la salud fisiológica y psicológica del poblador de Huepetuhe -Madre de Dios-2019
}

\author{
Viviana Patricia Aguirre Zender \\ vivianaaguirrezender@hotmail.com \\ Universidad Nacional Federico Villarreal, \\ Escuela Universitaria de Postgrado, Lima, Perú \\ Daniel Roberto Davila Revoredo \\ Universidad ESAN, Lima, Perú
}

\section{RESUMEN}

Objetivo: Determinar la influencia de la contaminación ambiental por mercurio en la salud fisiológica y psicológica del poblador de Huepetuhe-Madre de Dios, 2019. Investigación tipo aplicada; método descriptivo y nivel descriptivo - correlacional, transversal; diseño no experimental, técnica observacional y encuesta. Muestra: 200 pobladores; instrumento: Cuestionario con 30 preguntas para evaluar la contaminación ambiental por mercurio en la salud fisiológica y psicológica, con escala de Likert y juicio de expertos. Complementariamente, se valoraron concentraciones de mercurio en las muestras de músculo comestible (500 gr/cada especie) de cinco especies de pescado fresco de alto consumo en la zona de estudio (Mota punteada, chambira, doncella, zungaro, paiche) por medio de espectroscopía de emisión atómica, superaron los límites máximos permisibles de $0.5 \mathrm{mg} / \mathrm{kg}$ y $0.3 \mathrm{mg} / \mathrm{kg}$ permitidos por la Organización Mundial de la Salud y el US-EPA respectivamente. Conclusiones: Existe alta relación inversa entre la contaminación ambiental por mercurio con la salud fisiológica y psicológica del poblador de Huepetuhe. En las muestras de pescado analizadas se detectó contaminación por mercurio en todas las especies, y dos especies sobrepasaron niveles máximos permisibles, generando riesgo para la salud en los pobladores.

Palabras Claves: contaminación ambiental; mercurio, consumo de pescado contaminado; salud fisiológica y psicológica 


\title{
Environmental contamination by mercury and the physiological and psychological health of the resident of Huepetuhe -Madre de Dios-2019
}

\begin{abstract}
Objective: To determine the influence of environmental pollution by mercury on the physiological and psychological health of the resident of Huepetuhe-Madre de Dios, 2019. Applied type research; descriptive method and descriptive level - correlational, cross-sectional; non-experimental design, observational technique and survey. Sample: 200 residents; instrument: Questionnaire with 30 questions to evaluate environmental pollution by mercury in physiological and psychological health, with Likert scale and expert judgment. In addition, mercury concentrations were assessed in the edible muscle samples (500 gr / each species) of five species of fresh fish with high consumption in the study area (Mota punteada, chambira, maiden, zungaro, paiche) by means of spectroscopy. of atomic emission, exceeded the maximum permissible limits of $0.5 \mathrm{mg} /$ $\mathrm{kg}$ and $0.3 \mathrm{mg} / \mathrm{kg}$ allowed by the World Health Organization and the US-EPA respectively. Conclusions: There is a high inverse relationship between environmental contamination by mercury and the physiological and psychological health of the Huepetuhe resident. In the fish samples analyzed, mercury contamination was detected in all species, and two species exceeded maximum permissible levels, generating a health risk for the inhabitants.
\end{abstract}

Keywords: environmental pollution; mercury; consumption of contaminated fish; physiological and psychological health

Artículo recibido: 05 octubre. 2021 Aceptado para publicación: 02 noviembre 2021 Correspondencia: vivianaaguirrezender@hotmail.com Conflictos de Interés: Ninguna que declarar 


\section{INTRODUCCIÓN}

La contaminación ambiental por mercurio es uno de los principales problemas ambientales asociado a la salud pública a nivel mundial, debe ser identificada en forma oportuna y precoz por las diferentes instituciones, para determinar los factores asociados de la salud en los aspectos fisiológico y psicológico, y de esta manera, facilitar las medidas de control y prevención para una apropiada toma de decisiones por los responsables a nivel regional, nacional e internacional. En un estudio científico de "Concentraciones de mercurio en peces y seres humanos en Puerto Maldonado" realizada por Fernández y Gonzales, de la Universidad Carnegie y otros, se presentan los resultados de niveles peligrosos para la salud humana, indica que cuatro especies de peces de consumo por la población de Madre de Dios poseen niveles de contaminación que sobrepasan los niveles máximos aceptables por las normas internacionales para metales pesados y mercurio. Diversas investigaciones realizadas por organizaciones privadas y del estado pusieron en alerta a las autoridades sobre el consumo de varias especies de peces contaminadas con mercurio (Ministerio de la Producción 2016 comunicado $\mathrm{N}^{\circ}$ 004-2026-SANIPES/DSNPA). El Gobierno Regional declaró en emergencia once distritos de Madre de Dios por contaminación por mercurio. La actividad minera aurífera en este departamento no solo está afectando las reservas naturales, sino que también causa graves daños en la salud de la población. El Convenio de Minamata (del que Perú forma parte), entró en vigencia mundial a partir del 16 de agosto del 2017; por lo tanto, estamos comprometidos internacionalmente a proteger la salud humana y el medio ambiente al controlar el ciclo del mercurio. Esto incluye la prohibición de nuevas minas de mercurio, la eliminación de las existentes y la regulación del uso de este metal en la minería aurífera artesanal y en pequeña escala. En el 2020, se realizó el I Simposio Interamericano Sobre el Mercurio, Madre de Dios. Se presentó una ponencia denominada: "Minería Artesanal: Impacto del $\mathrm{Hg}$ en la salud del trabajador y su familia". "El riesgo de consumir peces contaminados con mercurio, está asociado con los fenómenos de: bioacumulación y de biomagnificación, que quiere decir que los músculos de los peces tienen fijación del mercurio. (Newman, 2015); Asimismo, el riesgo de uso de mercurio en amalgamación para purificar oro afecta a las familias que hacen la quema de las amalgamas en sus propias casas, (para evitar el robo de su metal); los vapores de mercurio emitidos en este proceso, les afecta el sistema 
endocrino, y fisiológico, los niños pueden ser afectados con bajo peso al nacer y con riesgos de retardo mental, por habitar en zonas de influencia de extracción de mercurio", (Gastañaga, 2020). Los efectos de la exposición al mercurio orgánico (metilmercurio o etilmercurio) son primariamente neurológicos. Otros efectos tóxicos pueden incluir reducción de la fertilidad, crecimiento inapropiado, anormalidades del comportamiento, perdida de la audición, reducción a respuestas inmunológica. (Departamento de salud de Nuevo México-USA, 2017). Altas dosis de mercurio pueden ser fatales para los humanos, pero también muy bajas dosis de mercurio dentro de compuestos alimenticios son altamente adversas a la salud particularmente al sistema nervioso, recientemente se han encontrado relaciones con los sistemas cardiovascular, inmunológico y reproductivo. (Health and Environment Alliance, 2017). Tras la inhalación o ingestión de distintos compuestos de mercurio o tras la exposición cutánea a ellos se pueden observar trastornos neurológicos y del comportamiento, con síntomas como temblores, insomnio, pérdida de memoria, efectos neuromusculares, cefalea o disfunciones cognitivas y motoras. En trabajadores expuestos durante varios años a niveles atmosféricos de al menos 20 microgramos/m3 de mercurio elemental se pueden observar signos subclínicos leves de toxicidad para el sistema nervioso central. Se han descrito efectos en los riñones que van de la proteinuria a la insuficiencia renal". (OMS, 2017) Por todo ello, es importante tomar en cuenta lo expuesto en líneas arriba para determinar los efectos de la exposición del mercurio que afecta al medio ambiente y la salud de la población; en mérito a ello es que se realizó el presente estudio sobre la contaminación ambiental por mercurio y la salud fisiológica y psicológica del poblador de Huepetuhe -Madre de Dios.

\section{MÉTODO}

La Investigación fue de tipo aplicada, el método fue de corte transversal, el diseño fue no experimental, y del nivel descriptivo-correlacional. El enfoque de la investigación fue cuantitativo, diseño no experimental transversal,

La población: Estuvo conformada por 6,042 habitantes del distrito de Huepetuhe, provincia de Manú del departamento de Madre de Dios, según censo nacional INEI2017. Se realizó un muestreo aleatorio simple con distribución uniforme teniendo en cuenta una prevalencia máxima a la exposición del $50 \%(\mathrm{p}=0.50)$. 
Muestra: Se utilizó una muestra probabilística aleatoria simple con tamaño de muestra finita al $95 \%$ de confianza y un máximo error de 0.068. el tamaño de muestra fue $\mathrm{n}=200$.

Se utilizó un cuestionario de dos partes: La primera parte: "Contaminación ambiental por exposición al mercurio" consta de 10 preguntas en escala de Likert, de las cuales 05 preguntas corresponden a la dimensión consumo de pescado contaminado con mercurio y 05 preguntas se refieren a la dimensión toxicidad por concentración de mercurio en pescados, respectivamente, en el cual se formularon preguntas, las cuales han sido elaboradas para verificar la validez de las hipótesis formuladas en el trabajo de investigación. La segunda parte: "Salud Fisiológica y Psicológica" consta de 20 preguntas en escala de Likert, de las cuales 10 preguntas corresponden a la dimensión fisiológica y 10 preguntas se refieren a la dimensión psicológica, respectivamente, las cuales han sido validadas por expertos. Se procedió a tabular toda la información mediante la creación de una base de datos utilizando para ello el SPSS versión 25.

\section{RESULTADOS}

\section{Descripción de los resultados}

El promedio de edad de los pobladores encuestados es de 33 años con una desviación estándar de \pm 12.77 años. La edad mínima fue de 18 años y la edad máxima fue de 84 años. Se tiene que el 64\% (128) eran de sexo Masculino y el 36\% (72) eran de sexo Femenino. Los pobladores encuestados tienen un tiempo de residencia promedio de 12 años aproximadamente. el 33.5\% (67) trabajan en actividad de extracción de oro y el $66.5 \%$ (133) no trabajan en extracción de oro. Los pobladores que se dedican a la extracción de oro, tienen un tiempo de actividad promedio de 7 años aproximadamente con una desviación estándar de \pm 7.89 años.

\section{Tabla 1}

Nivel de Contaminación ambiental por exposición al mercurio de los pobladores

\begin{tabular}{ccc}
\hline Contaminación Ambiental por Mercurio & Frecuencia & Porcentaje \\
\hline Baja & 28 & $14.00 \%$ \\
Regular & 109 & $54.50 \%$ \\
Alta & 63 & $31.50 \%$ \\
\hline Total & $\mathbf{2 0 0}$ & $\mathbf{1 0 0 . 0 0 \%}$ \\
\hline
\end{tabular}

Fuente: Elaboración propia

La tabla 1, muestra el nivel de la Contaminación ambiental por exposición al mercurio, $54.50 \%$ tienen un nivel Regular y el $31.50 \%$ tiene un nivel Alto, en el distrito de 
Huepetuhe del departamento de Madre de Dios.

\section{Tabla 2}

Nivel de consumo de pescado contaminado por mercurio

\begin{tabular}{ccc}
\hline Contaminación & Frecuencia & Porcentaje \\
\hline Baja & 33 & $16.50 \%$ \\
Regular & 97 & $48.50 \%$ \\
Alta & 70 & $35.00 \%$ \\
\hline Total & $\mathbf{2 0 0}$ & $\mathbf{1 0 0 . 0 0 \%}$ \\
\hline
\end{tabular}

Fuente: Elaboración propia

La tabla 2, Se puede establecer, que casi el $50 \%$ presentan un consumo regular y un $35 \%$ presentan un consumo alto de especies de pescado que están prohibidas por estar contaminados con mercurio en el distrito de Huepetuhe del departamento de Madre de Dios.

\section{Tabla 3}

Nivel de Toxicidad de los pobladores

\begin{tabular}{ccc}
\hline Toxicidad & Frecuencia & Porcentaje \\
\hline Baja & 24 & $12.00 \%$ \\
Regular & 85 & $42.50 \%$ \\
Alta & 91 & $45.50 \%$ \\
\hline Total & $\mathbf{2 0 0}$ & $\mathbf{1 0 0 . 0 0 \%}$
\end{tabular}

Fuente: Elaboración propia

La tabla 3, muestra la distribución de los pobladores según el nivel de Toxicidad, donde se observa que el $12 \%$ de los pobladores tienen un nivel Bajo, el $42.50 \%$ tienen un nivel Regular y el $45.50 \%$ tiene un nivel Alto, en el distrito de Huepetuhe del departamento de Madre de Dios.

\section{Tabla 4}

Nivel de Salud fisiológica y psicológica de los pobladores

\begin{tabular}{ccc}
\hline Salud fisiológica y psicológica & Frecuencia & Porcentaje \\
\hline Mala & 65 & $32.50 \%$ \\
Regular & 115 & $57.50 \%$ \\
Buena & 20 & $10.00 \%$ \\
\hline Total & $\mathbf{2 0 0}$ & $\mathbf{1 0 0 . 0 0 \%}$ \\
\hline
\end{tabular}

Fuente: Elaboración propia

La tabla 4, muestra la distribución de los pobladores según el nivel de Salud fisiológica y psicológica, donde se observa que el $32.50 \%$ de los pobladores tienen un nivel Malo, el $57.50 \%$ tienen un nivel Regular y el $10 \%$ tiene un nivel Bueno, en el distrito de Huepetuhe del departamento de Madre de Dios. 


\section{Tabla 5}

Nivel de Salud fisiológica de los pobladores

\begin{tabular}{ccc}
\hline Salud fisiológica & Frecuencia & Porcentaje \\
\hline Mala & 93 & $46.50 \%$ \\
Regular & 89 & $44.50 \%$ \\
Buena & 18 & $9.00 \%$ \\
\hline Total & $\mathbf{2 0 0}$ & $\mathbf{1 0 0 . 0 0 \%}$ \\
\hline
\end{tabular}

La tabla 5, muestra la distribución de los pobladores según el nivel de Salud fisiológica, donde se observa que el $46.50 \%$ de los pobladores tienen un nivel Malo, el $44.50 \%$ tienen un nivel Regular y el 9\% tiene un nivel Bueno, en el distrito de Huepetuhe del departamento de Madre de Dios.

\section{Tabla 6}

Nivel de Salud psicológica de los pobladores

\begin{tabular}{ccc}
\hline Salud psicológica & Frecuencia & Porcentaje \\
\hline Mala & 64 & $32.00 \%$ \\
Regular & 112 & $56.00 \%$ \\
Buena & 24 & $12.00 \%$ \\
\hline Total & 200 & $100.00 \%$ \\
\hline
\end{tabular}

Fuente: Elaboración propia

La tabla 6, muestra la distribución de los pobladores según el nivel de Salud psicológica, donde se observa que el $32 \%$ de los pobladores tienen un nivel Malo, el $56 \%$ tienen un nivel Regular y el 12\% tiene un nivel Bueno, en el distrito de Huepetuhe del departamento de Madre de Dios.

\section{Tabla 7}

Contaminación de Mercurio en peces en Huepetuhe - Madre de Dios. Abril - 2020

\begin{tabular}{cc} 
Peces & $\begin{array}{c}\text { Mercurio }(\mathbf{H g}) \\
(\mathbf{m g} / \mathbf{k g})\end{array}$ \\
\hline Mota Punteada & 1,26 \\
Chambira & 1,05 \\
Doncella & 0,30 \\
Paiche & 0,27 \\
Zumgaro & 0,21 \\
\hline
\end{tabular}

Fuente: Pruebas en Laboratorio ITS (Inspection\&Testing Services de Perú SAC). Del 06-03-2020 
Figura 1. Estadística de contaminación de mercurio en Huepetuhe-Madre de Dios.

Abril 2020

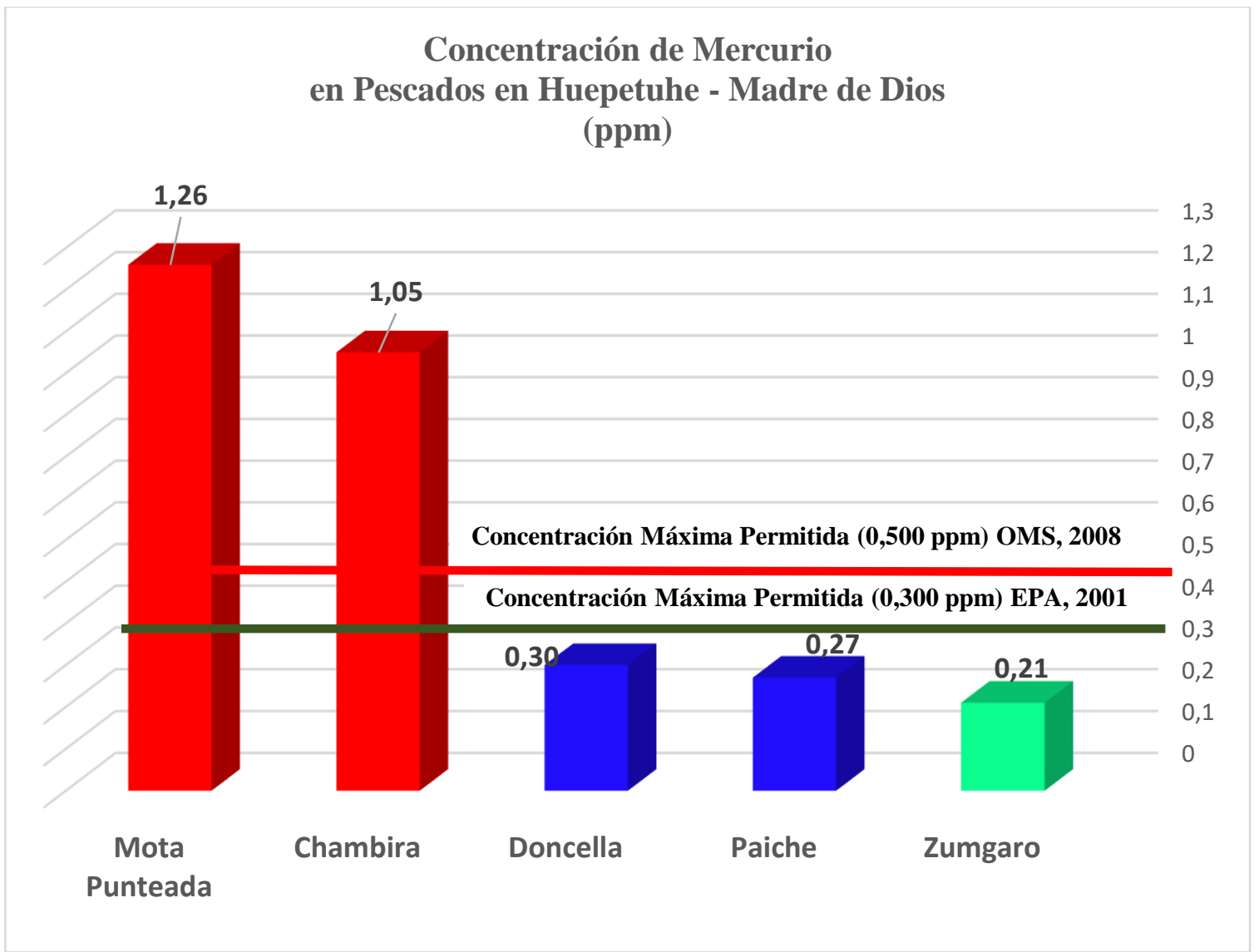

Fuente: Elaboración propia, a partir de pruebas en Laboratorio ITS (Inspection \& Testing Services de Perú SAC). ICP Optical, del 06-03-2020

De la tabla 7 y figura 1, muestra los niveles de concentración de mercurio en peces en Huepetuhe-Madre de Dios; donde se observa que las especies: Mota punteada (1,26 mg de $\mathrm{Hg}$ g/ Kg.), Chambira (1,05 mg de Hg/ Kg.), Doncella (0,30 mg de Hg/ Kg.), Paiche (0,27 mg de $\mathrm{Hg} / \mathrm{Kg}$. ), Zumgaro (0,21 mg de $\mathrm{Hg} / \mathrm{Kg}$.), expresan niveles de concentración de mercurio en sus tejidos, siendo el límite máximo permitido para consumo humano por la OMS (2008) de 0,50 mg de $\mathrm{Hg} / \mathrm{Kg}$., y, según el parámetro límite máximo de consumo permitido por la US-EPA (2001) de 0,30 mg de Hg/ Kg.y Mencionado por ONU (2008): por lo que se evidencia que todas las especies de peces muestreados, están contaminadas con mercurio, generando un riesgo para la salud en los pobladores. 


\section{DISCUSIÓN DE RESULTADOS}

- Existe una alta relación inversa entre la Contaminación Ambiental por Mercurio con la Salud fisiológica y psicológica de los pobladores de Huepetuhe en Madre de Dios.

De acuerdo a la tabla 1, muestra el nivel de la Contaminación ambiental por exposición al mercurio, el 54.50\% tienen un nivel Regular y el 31.50\% tiene un nivel Alto, en los pobladores del distrito de Huepetuhe del departamento de Madre de Dios. Dichos resultados son compatibles con los hallazgos de Panduro (2020), Vega (2019), Loza del Carpio A., Ccancapa Y. (2020).

Al respecto Panduro G. et. al. (2020) en su investigación: "Bioacumulación por mercurio en peces y riesgo por ingesta en una comunidad nativa en la amazonia peruana". Nos comenta que: El mercurio $(\mathrm{Hg})$ es un metal pesado tóxico que provoca daños en el sistema nervioso central, altera el comportamiento humano y provoca impacto en la biota acuática. El objetivo del presente trabajo fue evaluar la bioacumulación por $\mathrm{Hg}$ en peces y el riesgo por ingesta en una Comunidad Nativa (CN) en la amazonia peruana durante la estación seca y lluviosa. Se concluye que el riesgo a la salud del poblador de la comunidad nativa de la Amazonía peruana por el MeHg es alto.

Vega C. et. Al. (2019) en su investigación "Exposición elevada al mercurio realizada en una tribu de indígenas Machiguenga, en la zona del Manú, Madre de Dios”, indica: El enorme aumento de la minería prácticamente no regulada en Madre de Dios Perú está provocando una liberación masiva de mercurio elemental líquido al medio ambiente. Un análisis de regresión revela que el consumo de pescado, el género y el lugar de residencia fueron indicadores importantes de los niveles de mercurio; mientras que la duración de la residencia y la edad no tuvieron una relación significativa con los niveles de mercurio. El aumento de los niveles de consumo de pescado fue el indicador más fuerte del aumento de los niveles de mercurio total en toda la población.

Loza del Carpio A., Ccancapa Y. (2020) en su investigación sobre "Mercurio en un arroyo altoandino con alto impacto por minería aurífera artesanal (la rinconada, Puno, Perú)" Nos indica que; los índices de acumulación ratificaron que el lugar se encuentra extremadamente contaminado por mercurio, y representa un alto riesgo para la salud pública y los ecosistemas involucrados". 
De acuerdo a la tabla 3, muestra la distribución de los pobladores según el nivel de Toxicidad, donde se observa que el $12 \%$ de los pobladores tienen un nivel Bajo, el $42.50 \%$ tienen un nivel Regular y el $45.50 \%$ tiene un nivel Alto, en el distrito de Huepetuhe del departamento de Madre de Dios. Dichos resultados son compatibles con los hallazgos de Aparicio, L. (2015), Fields, Ch. et.al. (2017).

Aparicio, L. (2015) en su estudio: "El Mercurio En La Cuenca Del Tambopata. Repercusiones En La Salud Humana y Del Ecosistema" nos indica que: El objetivo de esta investigación fue determinar la presencia de mercurio-total en la cuenca del río Tambopata. Se colectó un total de 163 muestras de tejido de peces y cabello de seres humanos durante los años 2003, 2004 y 2005. Los resultados indican presencia de mercurio total en las partes alta y baja de la cuenca; se detectaron valores cercanos o que superan los límites recomendados por la OMS tanto para tejido de peces de consumo humano como en el cabello de los humanos analizados; significando un grave riesgo de neurotoxicidad para los sujetos y su descendencia.

Fields, Ch. et.al. (2017) "Persistencia de neurotoxicidad sensorial y motora inducida por mercurio: revisión sistemática de trabajadores previamente expuestos al vapor de mercurio". USA. Revisiones críticas en toxicología en 16 países. Indican que: El Mercurio elemental $(\mathrm{Hg} 0)$ es un neurotóxico bien reconocido, pero no se sabe si y por qué durante mucho tiempo persiste su neurotoxicidad.

De acuerdo a la tabla 4, muestra la distribución de los pobladores según el nivel de Salud fisiológica y psicológica, donde se observa que el $32.50 \%$ de los pobladores tienen un nivel Malo, el 57.50\% tienen un nivel Regular y el 10\% tiene un nivel Bueno, en el distrito de Huepetuhe del departamento de Madre de Dios. Dichos resultados son compatibles con los hallazgos de: (Baird y Cann, 2014).

"De acuerdo con la literatura revisada con respecto a la exposición del mercurio y sus efectos tóxicos en la salud del individuo, se asocian las siguientes alteraciones; a nivel del sistema neurológico, dermatológico, hormonal, renal, cardiológico y también a nivel psiquiátrico. Con lo ocurrido en el pueblo pesquero de Minamata Japón. En los años "50" miles de habitantes se contaminaron por mercurio a través de la ingesta de peces contaminados y cientos de ellos llegaron hasta morir. Estas personas presentaron los siguientes síntomas; Disfunción del sistema nervioso central (ya que el primer órgano 
en ubicarse el metilmercurio es el cerebro); dando lugar a la insensibilidad en brazos y piernas, visión borrosa, disminución de la visión, audición y coordinación muscular, letargo e irritabilidad”. (Baird y Cann, 2014).

- Existe una moderada relación inversa entre la Contaminación Ambiental por Mercurio con la Salud fisiológica de los pobladores de Huepetuhe en Madre de Dios.

De la tabla 5, muestra la distribución de los pobladores según el nivel de Salud fisiológica, donde se observa que el $46.50 \%$ de los pobladores tienen un nivel Malo, el $44.50 \%$ tienen un nivel Regular y el 9\% tiene un nivel Bueno, en el distrito de Huepetuhe del departamento de Madre de Dios. Dichos resultados son compatibles con los hallazgos de: Butscher F. et.al. (2020), El Ministerio de Salud y Protección Social (MSPS) Colombia, el Instituto Nacional de Salud (INS) y la Universidad de Córdoba (2017), (OPS, 2019), (ONU, 2017), (López y otros, 2016), CENSOPAS (2010).

Al respecto Butscher F. et.al. (2020); en su investigación "Calidad de vida relacionada con la salud $(\mathrm{EQ}-5 \mathrm{D}+\mathrm{C})$ entre las personas que viven en áreas de extracción de oro artesanal y en pequeña escala en Zimbabwe: un estudio transversal" señala lo siguiente: que 500.000 personas trabajan en el sector de la minería de oro artesanal. El uso de mercurio es común para extraer oro del mineral. La exposición prolongada al mercurio puede causar diversas condiciones de salud adversas, incluida la intoxicación crónica por mercurio. Aún se desconoce la influencia de estos efectos adversos para la salud en la calidad de vida relacionada con la salud (CVRS), se recogió muestras humanas (sangre, orina). Resultados: Los 207 participantes (82\% hombres, edad media 38 años) informaron 40 estados de salud diferentes. De los participantes del estudio, el 42,5\% informó estar completamente bien de salud, mientras que el 57,5\% informó que se encontraba mal de diferentes maneras. Nueve participantes $(4,3 \%)$ fueron identificados con intoxicación crónica por mercurio, mientras que 92 participantes $(33,3 \%)$ tenían niveles de mercurio por encima del umbral de "Alerta" en al menos una muestra.

El Ministerio de Salud y Protección Social (MSPS) Colombia, el Instituto Nacional de Salud (INS) y la Universidad de Córdoba (UC), desarrollaron un estudio (2017), que tuvo como objeto identificar los efectos sobre la salud por exposición a mercurio en departamentos priorizados con presencia de minería aurífera y sus impactos, entre los departamentos analizados se incluyó Chocó y es de este que se presentan los hallazgos. 
Se incluyeron en el estudio 1096 individuos de 11 municipios. Se encontró que el tiempo promedio de exposición a mercurio en sus labores fue de 18 años y el tiempo promedio de uso de 4 años. Se tomaron muestras en cualquiera de las tres matrices biológicas evaluadas (sangre, orina y cabello). El 47,3 \% (519) de las personas cumplieron con la definición de caso de persona intoxicada con mercurio establecida por el INS. Estas personas fueron remitidas a sus EPS, para ser canalizadas a la ruta de atención en salud y así determinar si requerían tratamiento médico. A partir del enfoque de género se lograron percibir y comprender las preocupaciones y experiencias de mujeres y hombres frente a la minería. En materia de salud la minería es percibida, como una de las principales causas de afectación de la salud de la población chocoana causante de enfermedades como el cáncer, el deterioro de órganos, alteraciones en el sistema nervioso, malformaciones fetales, intoxicación y muerte.

La Organización Panamericana de la Salud indica que: "El mercurio es tóxico para la salud humana y representa una amenaza particular para el desarrollo del niño en el útero y temprano en la vida. El mercurio existe en varias formas: elemental (o metálico); inorgánico (por ejemplo, cloruro mercúrico); y orgánicos (p. ej., metil y etilmercurio); todas tienen efectos tóxicos, incluso en los sistemas nervioso, digestivo e inmunitario, y en los pulmones, los riñones, la piel y los ojos”. (OPS, 2019)

"El mercurio elemental y el metilmercurio son tóxicos para el sistema nervioso central y el periférico. La inhalación de vapor de mercurio puede ser perjudicial para los sistemas nervioso e inmunitario, el aparato digestivo y los pulmones y riñones, con consecuencias a veces fatales. Las sales de mercurio inorgánicas son corrosivas para la piel, los ojos y el tracto intestinal y, al ser ingeridas, pueden resultar tóxicas para los riñones". (ONU, 2017)

En un estudio se "examina los efectos en la salud del individuo y su relación con la actividad minera, mediante la exposición a contaminantes tóxicos determinados como factores de riesgo para los mineros, población del cantón Portovelo provincia de El OroEcuador. (...) Los resultados revelan problemas de salud que afectan al aparato locomotor, sistema nervioso y problemas congénitos. Su severidad está dada por la dosis, la edad, la duración, las vías de exposición, y factores ambientales, nutricionales y genéticos" (López y otros, 2016)

En referencia a los síntomas asociados a la exposición a mercurio, Al respecto 
CENSOPAS Centro Nacional de Salud Ocupacional y Protección del Ambiente para la Salud (2010), en su estudio: “Niveles de exposición a mercurio en población de Huepetuhe-Madre de Dios y Factores de Riesgo de Exposición, nos indican que, de los $31.2 \%$ de los evaluados refirieron debilidad muscular, $12.7 \%$ temblores, $37.7 \%$ dolor de cabeza, $22.3 \%$ alergias y $15.1 \%$ descamación de piel”.

- Se comprobó que existe una moderada relación inversa entre la Contaminación Ambiental por Mercurio con la Salud psicológica de los pobladores de Huepetuhe en Madre de Dios.

De acuerdo a la tabla 6, muestra la distribución de los pobladores según el nivel de Salud psicológica, donde se observa que el 32\% de los pobladores tienen un nivel Malo, el $56 \%$ tienen un nivel Regular y el $12 \%$ tiene un nivel Bueno, en el distrito de Huepetuhe del departamento de Madre de Dios. Dichos resultados son compatibles con los hallazgos de: Fields, Ch. et.al. (2018), (ONU, 2017), (Rodríguez, (2017), (MINSA,2015), CENSOPAS (2010).

Al respecto Fields, Ch. et.al. (2018), en su estudio "Neurotoxicidad sensorial y motora inducida por mercurio: revisión sistemática de trabajadores actualmente expuestos al vapor de mercurio", los resultados fueron los siguientes: Se identificaron cuarenta y cinco estudios elegibles, que incluían a más de 3000 trabajadores expuestos crónicamente a un rango de concentraciones de $\mathrm{Hg} 0$ (0,002 a 1,7 mg / m 3). Los efectos que demostraron coherencia entre los estudios y una mayor frecuencia en los niveles de mercurio en orina $(<50 ; 50-100 ; 100-200 ; \geq 200 \mu \mathrm{g} / \mathrm{L})$ incluyeron temblor, alteración de la coordinación y reflejos anormales en la EP, y rendimiento reducido en las pruebas NB de temblor, destreza manual y velocidad del motor.

La ONU nos manifiesta lo siguiente; "Tras la inhalación o ingestión de distintos compuestos de mercurio o tras la exposición cutánea a ellos se observan trastornos neurológicos y del comportamiento, con síntomas como temblores, insomnio, pérdida de memoria, efectos neuromusculares, cefalea o disfunciones cognitivas y motoras". (ONU, 2017).

En una investigación realizada en Cuba nos refiere: "Las intoxicaciones con mercurio pueden provocar alteraciones psicológicas. Las exposiciones leves a los vapores están caracterizadas por pérdida de la memoria, temblores, inestabilidad emocional (angustia e irritabilidad), insomnio. A exposiciones moderadas, se observan desórdenes mentales 
y perturbaciones motoras". (Rodríguez, 2017).

"El MINSA nos reporta con respecto a la intoxicación crónica por los vapores de mercurio las siguientes alteraciones psicológicas: Irritabilidad, tristeza, insomnio, ansiedad, pérdida de memoria, timidez, labilidad emocional, alteración del juicio, depresión e incluso estados paranoides. Esta sintomatología constituye el denominado “eretismo mercurial”. (MINSA,2015).

En referencia a los síntomas asociados a la exposición a mercurio, Al respecto CENSOPAS Centro Nacional de Salud Ocupacional y Protección del Ambiente para la Salud (2010), en su estudio: Niveles de exposición a mercurio en población de Huepetuhe-Madre de Dios y Factores de Riesgo de Exposición. Nos indican que, de los $31.2 \%$ de los evaluados refirieron pérdida de memoria, $29.5 \%$ cambio de ánimo, $24.3 \%$ irritabilidad.

De acuerdo a la tabla 14, muestra los niveles de concentración de mercurio en peces en Huepetuhe-Madre de Dios; donde se observa que las especies: Mota punteada (1,26 mg de $\mathrm{Hg}$ g/ Kg.), Chambira (1,05 mg de $\mathrm{Hg} / \mathrm{Kg}$.), Doncella (0,30 mg de Hg/ Kg.), Paiche (0,27 mg de $\mathrm{Hg} / \mathrm{Kg}$.), Zumgaro (0,21 mg de $\mathrm{Hg} / \mathrm{Kg}$.), expresan niveles de concentración de mercurio en sus tejidos, siendo el límite máximo permitido para consumo humano por la OMS (2008) de 0,50 mg de $\mathrm{Hg} / \mathrm{Kg}$., y, según el parámetro límite máximo de consumo permitido por la US-EPA (2001) de 0,30 mg de $\mathrm{Hg} / \mathrm{Kg}$.: por lo que se evidencia que todas las especies de peces muestreados, están contaminadas con mercurio, generando un riesgo para la salud en los pobladores. Dichos resultados son compatibles con los hallazgos de: Ninaja P. y Ortiz R. (2020), Fernández L. (2013), Vega C. et. Al. (2019).

Ninaja P. y Ortiz R. (2020) En su investigación realizada en Tacna-Perú," Niveles de mercurio en los pescados de mayor consumo en los mercados de Tacna exceden límites permitidos por entidades regulatorias", nos indican que; Según los límites máximos permisibles de $0.5 \mathrm{ppm}$ permitidos por la Organización Mundial de la Salud y la Agencia de Protección Ambiental de los Estados Unidos, también de 0.5 ppm; el 73.30 $\%$ de las muestras de pescado analizadas excedieron este parámetro de mercurio, generando un verdadero riesgo para la salud.

Fernández L. y otros (2013) en su investigación sobre: "Mercurio en Madre de Dios: Concentraciones de mercurio en peces y humanos en Puerto Maldonado". Indica que: 
Se analizaron concentraciones de mercurio en el tejido muscular de 15 especies de pescado comúnmente consumido, capturado en el medio silvestre y comprado en varios mercados de Puerto Maldonado durante agosto de 2012. El proyecto CAMEP ofreció pruebas gratuitas de mercurio en cabello, a una muestra de 226 personas adultas en Puerto Maldonado desde mayo hasta agosto de 2012. Resultados: -Los altos niveles de mercurio en el medio ambiente de Madre de Dios están afectando fuertemente al ser humano en la población de Puerto Maldonado, resultando una amenaza para la salud pública. -Los Niveles de mercurio de la población de Puerto Maldonado son extremadamente elevados y la contaminación por mercurio es más extensa de lo previamente identificado. -La contaminación por mercurio del pescado capturado en los ríos y vendido en los mercados de Puerto Maldonado está aumentando, lo que indica que los ecosistemas de Madre de Dios están siendo gravemente afectados por la minería artesanal de oro. -El consumo regular de especies silvestres de pescados capturados están contaminados con altas concentraciones de mercurio.

Vega C. et. Al. (2019) en su investigación "Exposición elevada al mercurio realizada en una tribu de indígenas Machiguenga, en la zona del Manú, Madre de Dios”, Un análisis de regresión revela que el consumo de pescado, el género y el lugar de residencia fueron indicadores importantes de los niveles de mercurio.

\section{CONCLUSIONES}

1) Existe una alta relación inversa entre la Contaminación Ambiental por Mercurio con la Salud fisiológica y psicológica de los pobladores del distrito de Huepetuhe en el departamento de Madre de Dios. Cabe destacar que, si se reducen los niveles de contaminación ambiental por mercurio en el distrito de Huepetuhe, los niveles de salud fisiológica y psicológica de los pobladores mejorarían.

2) Existe una moderada relación inversa entre la Contaminación ambiental por exposición al mercurio con la Salud fisiológica de los pobladores del distrito de Huepetuhe en el departamento de Madre de Dios. Cabe destacar, que, si se reducen los niveles de contaminación ambiental por exposición al mercurio en el distrito de Huepetuhe, los niveles de salud fisiológica de los pobladores mejorarían.

3) Existe una moderada relación inversa entre la Contaminación ambiental por exposición al mercurio con la Salud psicológica de los pobladores del distrito de Huepetuhe en el departamento de Madre de Dios. Cabe destacar, que, si se reducen 
los niveles de Contaminación ambiental por exposición al mercurio en el distrito de Huepetuhe, los niveles de salud psicológica de los pobladores mejorarían.

\section{RECOMENDACIONES}

1) Fomentar la investigación científica con respecto a la contaminación ambiental por mercurio y sus efectos fisiológicos, psicológicos, así como, también sociales y económicos.

2) Desarrollar programas de monitoreo sobre contaminación ambiental por mercurio a corto, mediano y largo plazo; así como estrategias de control y planes de prevención de la salud pública y ambiental a nivel nacional e internacional.

3) 3. Proponer programas sociales poblacionales de cambio de actitud y percepción con respecto al consumo de peces contaminados en la región, ya que a pesar de que tienen conocimiento sobre los niveles de contaminación, la población continúa consumiendo los peces de especies contaminadas.

4) Realizar futuros estudios sobre Contaminación ambiental por mercurio y la salud fisiológica y psicológica, sustentados mediante análisis y monitoreo de niveles de mercurio en peces contaminados y análisis de cabello, sangre y orina; de niveles de mercurio, para los pobladores de las zonas de influencia.

\section{REFERENCIAS BIBLIOGRÁFICAS}

Aparicio, L. (2015) "El Mercurio En La Cuenca Del Tambopata. Repercusiones En La Salud Humana y Del Ecosistema" Universidad Nacional Agraria La Molina. Recuperado de https://core.ac.uk/download/pdf/162860597.pdf

Arana M. (2009) "El caso del derrame de mercurio en Choropampa y los daños a la salud en la población rural expuesta" Rev. Perú Med. Exp. Salud Publica.; 26(1): 113-18. Recuperado de https://www.ins.gob.pe/insvirtual/images/artrevista/pdf/rpmesp2009.v26.n1.a1 9.pdf

Artisanal Gold Council.(2017) ONG de Investigación Medioambiental. Recuperado de https://www.artisanalgold.org/wordpress/wp-content/uploads/2020/09/Reportede-inventario-MAPE_Peru.pdf

Baird C. y Cann M. (2014) Química Ambiental. Metales Pesados Tóxicos Cap. 12. Segunda edición. Editorial Reverté, Barcelona, pp.519-536. 
Butscher F. et.al. (2020) "Calidad de vida relacionada con la salud (EQ- 5D + C) entre las personas que viven en áreas de extracción de oro artesanal y en pequeña escala en Zimbabwe: un estudio transversal". Recuperado de https://www.researchgate.net/publication/343726596_Healthrelated_quality_of_life_EQ-

5D_C_among_people_living_in_artisanal_and_smallscale_gold_mining_areas_in_Zimbabwe_a_cross-sectional_study

CENSOPAS Centro Nacional de Salud Ocupacional y Protección del Ambiente para la Salud (2010) "Niveles de exposición a mercurio en población de HuepetuheMadre de Dios y Factores de Riesgo de Exposición 2012. Recuperado de http://mddconsortium.org/wp-content/uploads/2014/11/CENSOPAS-2010-

Niveles-de-exposicion-a-mercurio-en-poblacion-de-Huepetuhe-Madre-de-

Dios.pdf

CINCIA, (2017) "Prevención de exposición por mercurio. Guía para promotores". Wake Forest University. Recuperado de http://cincia.wfu.edu/wpcontent/uploads/Guia-del-Promotor-Prevencion-mercurio-03-17.pdf

Departamento de salud de Nuevo México (2017) "Mercury in the Environment and Health Effects". Recuperado de https://www.env.nm.gov/dwb/contaminants/documents/MercuryFactSheet.pdf

Diaz-Arriaga (2014) "Mercurio en la minería del oro: impacto en las fuentes hídricas destinadas para consumo humano", Revista Salud Pública. Recuperado de http://www.scielosp.org/pdf/rsap/v16n6/v16n6a12.pdf

Encinas M. (2011) "Medio Ambiente y Contaminación. Principios básicos", 1ra Edición. https://addi.ehu.es/bitstream/handle/10810/16784/Medio\%20Ambiente\%20y\% 20Contaminaci\%C3\%B3n.\%20Principios $\% 20 \mathrm{~b} \% \mathrm{C} 3 \% \mathrm{~A} 1$ sicos.pdf?sequence $=$ $\underline{6}$.

EPA Agencia de Protección Ambiental de Estados Unidos (2018) "Efectos en la salud por la exposición al mercurio". Recuperado de https://espanol.epa.gov/espanol/efectos-en-la-salud-por-la-exposicion-al$\underline{\text { mercurio }}$ 
Faial, K. y Deus, S. (2018). "Flujo de deposición de mercurio y selenio encabellos de ribereños en habitantes del Amazonas, Brasil. ITEGAM-JETIA , 4 (14), 32-45. Recuperado de https://itegam-jetia.org/journal/index.php/jetia/article/view/27

Fernandez L. (2013) "Mercurio en Madre de Dios. Concentraciones de mercurio en peces y seres humanos en Puerto Maldonado”, CAMEP Carnegie Amazonic Mercury Ecosystem Project. Recuperado de https://wwwlegacy.dge.carnegiescience.edu/research/CAMEP/CAMEP\%20Research\%20B rief\%20-\%20Puerto\%20Maldonado\%20English\%20-\%20FINAL.pdf

Fields, Ch. et.al. (2018) "Neurotoxicidad sensorial y motora inducida por mercurio: revisión sistemática de trabajadores actualmente expuestos al vapor de mercurio", Biblioteca Nacional de Estados Unidos. PNC. Recuperado de https://www.ncbi.nlm.nih.gov/pmc/articles/PMC5931703/

Fields, Ch. et.al. (2017) "Persistencia de neurotoxicidad sensorial y motora inducida por mercurio: revisión sistemática de trabajadores previamente expuestos al vapor de mercurio". USA. Revisiones de 16 países, críticas en toxicología. Recuperado de https://www.researchgate.net/publication/318547783_Persistence_of_mercuryinduced_motor_and_sensory_neurotoxicity_systematic_review_of_workers_pr eviously_exposed_to_mercury_vapor

Gaona X. (2004) "El mercurio como contaminante global, Universidad politécnica de Catalunya, Departamento de Química, España”. Recuperado de https://www.tdx.cat/handle/10803/3174\#page=1

Gastañaga M. (2020) "Minería Artesanal: Impacto del Hg en la salud del trabajador y su familia”. I Simposium Interamericano Sobre el Mercurio, Madre de Dios. Recuperado de https://www.youtube.com/watch?v=bgdabz-9QGs

Global Environment Fund (GEF , 2017). Recuperado de https://www.retema.es/noticia/convenio-de-minamata-128-paises-se-unencontra-la-contaminacion-por-mercurio-7xjPA

Gómez, Marcelo M. (2006). Introducción a la Metodología de la Investigación Científica. Córdoba, Argentina. Edit. Brujas 
Gómez, C. (2012) Ecología Aplicada "Evaluación de Escenarios Alternativos en Sistemas Social Ecológicos Afectados por la Minería Aluvial en Madre de Dios", Universidad Agraria de La Molina. Lima, Perú. Recuperado de file:///C:/Users/Cliente/Downloads/gomez_ac\%20(2).pdf.

GOREMAD (2013) Gobierno Regional de Madre de Dios, Programa Regional de población de la Región Madre de Dios 2013-2017, Gerencia de Desarrollo Social. Recuperado de https://www.mimp.gob.pe/webs/mimp/sispod/PROGRAMAS\%20REGIONAL ES/MadredeDios/PROG_MADREDEDIOS.pdf.

Health and Environment Alliance (2017). Recuperado de http://www.envhealth.org/IMG/pdf/mercury_chapter1.pdf

Hernández, R. Fernández-Collado, C. Babtista P. (2014). Metodología de la Investigación. Sexta edición p. 158. Bogotá. MC Graw- Hill.

Higueras P. (2019) "Mercurio y consumo de pescado", Universidad de Castilla-La Mancha, España. Recuperado de https://theconversation.com/mercurio-yconsumo-de-pescado-120630

López M. et. Al. (2016) “Actividad minera y su impacto en la salud humana”, Revista Ciencia UNEMI. Recuperado de file:///C:/Users/DANIEL\%20DAVILA/Downloads/DialnetActividadMineraYSuImpactoEnLaSaludHumana-5556797.pdf

Loza del Carpio A., Ccancapa Y. (2020) "Mercurio en un arroyo altoandino con alto impacto por minería aurífera artesanal (la rinconada, Puno, Perú)“ Revista Int. Contaminación Ambiental. Recuperado de https://app.ingemmet.gob.pe/biblioteca/pdf/Amb-259.pdf

MINAM (2011), Ministerio del Ambiente, Minería aurífera en Madre de Dios y contaminación con mercurio, una bomba de tiempo". Recuperado de http://siar.minam.gob.pe/puno/sites/default/files/archivos/public/docs/mineria aurifera_en_madre_de_dios.pdf

MINAM (2017), Ministerio del Ambiente, Por la ratificación del convenio de Minamata, Perú. Recuperado de http://www.minam.gob.pe/wpcontent/uploads/2015/10/cuadernillo_minamata.pdf. 
MINAM (2019), Ministerio del Ambiente, Plan Nacional de Aplicación del Convenio de Minamata sobre el Mercurio, Perú. Recuperado de https://sinia.minam.gob.pe/normas/aprueban-plan-nacional-aplicacionconvenio-minamata-mercurio

MINSA (2015) “Guía de práctica clínica para el diagnóstico y tratamiento de la intoxicación por Mercurio”. Perú. Recuperado de http://bvs.minsa.gob.pe/local/MINSA/3245.pdf

Ministerio de Salud y Protección Social MSPS Colombia, Universidad de Córdoba (UC), Instituto Nacional de Salud (INS). (2016) "Evaluación epidemiológica de los efectos en salud por exposición ocupacional y ambiental a mercurio en el departamento de Chocó”. Recuperado de https://www.minsalud.gov.co/sites/rid/Lists/BibliotecaDigital/RIDE/VS/PP/SA /protocolo-sentencia-t622-vcolciencias.pdf

Mosquera C. y otros (2009) "Estudio Diagnóstico de la actividad Minera Artesanal en Madre de Dios". Recuperado de http://www2.congreso.gob.pe/sicr/cendocbib/con4_uibd.nsf/3CD1F208E6B9E C6D05257C1A006FF9C0/\$FILE/estudio_diagnostico_mineria_artesanal_madr edios.pdf

Newman M. (2015) Fundamentals of Ecotoxicology: The Science of Pollution. Fourth Edition. CRC Press. New York. Recuperado de https://www.academia.edu/36872120/Newman_Michael_C_Fundamentals_of Ecotoxicology_The_Science_of_Pollution_2014_CRC_Press

Mosquera C. et. al. (2009) "Estudio Diagnóstico de la actividad Minera Artesanal en Madre de Dios Recuperado de http://www2.congreso.gob.pe/sicr/cendocbib/con4_uibd.nsf/3CD1F208E6B9E C6D05257C1A006FF9C0/\$FILE/estudio_diagnostico_mineria_artesanal_madr edios.pdf

Ninaja P. y Ortiz R. (2020)," Niveles de mercurio en los pescados de mayor consumo en los mercados de Tacna exceden límites permitidos por entidades regulatorias". Revista Médica Basadrina, Recuperado de http://webcache.googleusercontent.com/search?q=cache:6QyhEJWgSG4J:revis tas.unjbg.edu.pe/index.php/rmb/article/download/922/1033/+\&cd=1\&hl=es- 
$\underline{419 \& \mathrm{ct}=\mathrm{clnk} \& \mathrm{gl}=\mathrm{pe}}$

OMS Organización Mundial de la Salud (2006) "Documentos básicos, suplemento de la 45a edición, octubre de 2006- CONSTITUCION DE LA OMS. Recuperado de http://www.who.int/governance/eb/who_constitution_sp.pdf

OMS Organización Mundial de la Salud (2008) Guidance for Identifying Populations at Risk from Mercury Exposure. Recuperado de https://www.who.int/foodsafety/publications/chem/mercuryexposure.pdf

OMS Organización Mundial de la Salud (2017) "El mercurio y la salud”. Recuperado de https://www.who.int/es/news-room/fact-sheets/detail/mercury-and-health

OMS Organización Mundial de la Salud (2013) "Efectos de la exposición al mercurio en la salud de las personas que viven en comunidades donde se practica la minería aurífera artesanal y en pequeña escala”. Recuperado de http://www.who.int/ipcs/assessment/public_health/mercury_asgm_es.pdf.

ONU (2017) Naciones Unidas Para el Medioambiente. Recuperado de http://www.unep.org/newscentre/es/el-mundo-se-une-contra-lacontaminaci\%C3\%B3n-por-mercurio-con-la-entrada-en-vigor-del-conveniode.

OPS (2019) Organización Mundial de la Salud- Panamerican Health Organization. Recuperado de https://webcache.googleusercontent.com/search?q=cache:y6D9srwPiRYJ:https ://www.paho.org/es/temas/seguridad-quimica/mercurio+\&cd=11\&hl=es$\underline{419 \& \mathrm{ct}=\mathrm{clnk} \& \mathrm{gl}=\mathrm{pe}}$

Osores F., Rojas J., Manrique C. (2012) "Minería informal e ilegal y contaminación con mercurio en Madre de Dios: Un problema de salud pública". Revista Acta Medica Peruana. Recuperado de https://www.researchgate.net/publication/262628006_Mineria_informal_e_ileg al_y_contaminacion_con_mercurio_en_Madre_de_Dios_Un_problema_de_sal ud_publica

Panduro G. et. al. (2020) "Bioacumulación por mercurio en peces y riesgo por ingesta en una comunidad nativa en la amazonia peruana”. Recuperado de https://www.researchgate.net/publication/343365711_Bioacumulacion_por_me rcurio_en_peces_y_riesgo_por_ingesta_en_una_comunidad_nativa_en_la_ama 
$\underline{\text { zonia_peruana }}$

Quintero, C.; Fonticiella E. (2012) " Algunas consideraciones filosóficas sobre fundamentos filosóficos de los problemas del medio ambiente". Revista Desarrollo Local Sostenible. Recuperado de http://www.eumed.net/rev/delos/14/qdfi.pdf

Ramírez A. (2008) "Intoxicación ocupacional por mercurio" Anales de la Facultad de medicina. Revista de Investigación UNMSM 69(1):46-51 Recuperado de http://revistasinvestigacion.unmsm.edu.pe/index.php/anales/article/view/1184.

Ruiz Peyré F., y Sosa C. (2019) “Apropiación diferencial de recursos naturales, acaparamiento y conflictos sociales en América Latina", "Convenio de Minamata: ¿Oportunidad o amenaza para la minería de oro a pequeña escala en el Perú?”, UniRío Editora, tomado de Capítulo 9 pp:194-225. Recuperado de http://www.unirioeditora.com.ar/wp-content/uploads/2019/08/978-987-688352-8.pdf

Rodríguez D. (2017) “Intoxicación ocupacional por metales pesados” MEDISAN vol.21 no.12 Santiago de Cuba. Recuperado de http://scielo.sld.cu/scielo.php?script=sci_arttext\&pid=S1029$\underline{30192017001200012}$

SANIPES Organismo Nacional de Sanidad Pesquera, (2016) “Comunicado 004-2016SANIPES/DSPA, Prohibición de Comercialización de Mota Punteada en Madre de Dios". Recuperado de http://www.sanipes.gob.pe/documentos/5_COMUNICADO-N-004-2016SANIPES-DSNPA.pdf

Turizio A. (2016). " Determinación de los niveles de mercurio en el distrito minero de San Martín de Loba, sur de Bolívar (Colombia)". Recuperado de https://repositorio.unicartagena.edu.co/handle/11227/3770

Vega C. et. al (2019) "Exposición elevada al mercurio en poblaciones indígenas amazónicas en el Parque Nacional Manu, Madre de Dios, Perú”. WFU, CINCIA-Madre de Dios Perú. Recuperado de https://www.researchgate.net/publication/340817007_Elevated_mercury_expos ure in_Amazonian_indigenous_populations_in_Manu_National_Park_Madre $\underline{\text { de_Dios_Peru }}$ 